\title{
Bipolar pulse coding for enhanced performance in Brillouin distributed optical fiber sensors
}

\author{
Marcelo A. Soto ${ }^{* a}$, Sébastien Le Floch ${ }^{\mathrm{b}}$, Luc Thévenaz ${ }^{\mathrm{a}}$ \\ ${ }^{a}$ EPFL Swiss Federal Institute of Technology, Institute of Electrical Engineering, \\ SCI STI LT, Station 11, CH-1015 Lausanne, Switzerland; \\ ${ }^{\mathrm{b}} \mathrm{HE}-\mathrm{ARC}$, Rue de la Serre 7, CH-2610 Saint Imier, Switzerland \\ *E-mail: marcelo.soto@epfl.ch
}

\begin{abstract}
We propose and experimentally demonstrate the possibility to use a pump signal based on bipolar pulse sequences using single-sideband suppressed-carrier (SSB-SC) modulation in Brillouin optical time-domain analysis (BOTDA) sensors. The SSB-SC modulated pump makes a sequential use of the Brillouin gain and loss spectra, increasing the intensity contrast of the measurements. The method is demonstrated using bipolar Golay codes along a $50 \mathrm{~km}$ sensing fiber and $2 \mathrm{~m}$ spatial resolution. Results indicate that the use of bipolar sequences provides a higher SNR enhancement and stronger robustness to pump depletion in comparison to BOTDA systems employing conventional unipolar sequences.
\end{abstract}

Keywords: Stimulated Brillouin scattering, distributed optical fiber sensor, temperature sensing, strain sensing

\section{INTRODUCTION}

Distributed optical fiber sensing based on stimulated Brillouin scattering (SBS) is a widely-used technique to measure distributed temperature and strain along several tens of km with meter-scale spatial resolution. The conventional timedomain approach, so called Brillouin optical time-domain analysis (BOTDA) $)^{1}$, uses a pulsed pump signal and a counterpropagating probe wave, which interact through mediation of an acoustic wave generated in the fiber through SBS. By sweeping the frequency detuning between optical waves, the Brillouin gain spectrum (BGS) as a function of the distance can be scanned with a spatial resolution fixed by the pulse width: the shorter the pulse width, the better is the spatial resolution. However, the weak SBS process leads to measurements with low SNR, mostly when long sensing ranges are involved, since the pump peak power cannot be increased indefinitely as a result of the onset of other nonlinear effects.

During the past few years, methods such as distributed Raman amplification ${ }^{2}$ and optical pulse coding ${ }^{3}$ have been proposed to increase the sensing distance of BOTDA sensors, reaching ranges beyond $100 \mathrm{~km}$ with meter-scale spatial resolution. While the former method requires the use of high-power lasers to produce distributed optical amplification along the fiber ${ }^{2}$, the latter technique increases the signal-to-noise ratio of the measurements by launching a sequence of pulses into the fiber, followed by a data processing procedure to retrieve the single-pulse response of the fiber ${ }^{3}$. The coding spreads the energy over many pulses, but the response after processing is equivalent to a single pulse containing the summed energy of all pulses in the sequence.

Known coding methods suitable for BOTDA applications employ unipolar (binary) pulse sequences, in which bits ' 0 s' and ' $1 \mathrm{~s}$ ' represent the ON-OFF status of the light ${ }^{3,4}$, and hence, they can be easily implemented using standard intensity modulation. However, the acoustic wave decay time imposes some restrictions to standard coding methods, since pulses in a sequence can easily interact with pre-existing acoustic waves activated by preceding pulses in the same sequence 5 To avoid distortions and maintain a linear Brillouin amplification, pulse coding has to be implemented using return-tozero (RZ) format ${ }^{5}$, allowing the acoustic wave amplitude to decay before other pulses are transmitted through the fiber.

In this paper we propose a new method to modulate the pump signal in BOTDA sensors. The technique is based on the combination of intensity-RZ pulse sequences with single-sideband suppressed-carried (SSB-SC) modulation, following a pattern described by bipolar pulse sequences. The bipolar pulses make use of Brillouin gain and loss processes, mitigating the impact of pump depletion and increasing the intensity contrast of the measured BGS, while optimizing the energy in the sequence since all pulses are ON. Here we unambiguously demonstrate the feasibility of the technique using a complementary-correlation pair of bipolar Golay codes in a $50 \mathrm{~km}$ BOTDA sensor with $2 \mathrm{~m}$ spatial resolution, but the method can be perfectly adapted to any suitable bipolar codes. 


\section{SSB-SC MODULATION BASED ON BIPOLAR CODES FOR BOTDA SENSORS}

The limitations imposed to the sensing distance when short pump pulses are used (to provide high spatial resolution) have been partially overcome using optical pulse coding methods ${ }^{3-5}$, in which a large number of pulses are launched into the fiber, avoiding the use of high peak power levels that could potentially induce detrimental nonlinear effects. In this way, the intensity contrast of the BOTDA traces is increased, leading to measurements with enhanced SNR.

Optical coding techniques for sensing applications are based on sequences of pulses which are intensity modulated according to specific unipolar (binary) codes, such as Simplex ${ }^{3}$ or Golay ${ }^{4}$ codes. These two coding methods have been found to be optimum when intensity pulses are used, providing practically the same SNR enhancement when using a large number of bits. The coding gain (which correspond to the SNR improvement) actually depends on the number of bits used in the sequences, and is given for such binary codes by: $C_{\text {gain }}=\sqrt{L} / 2$, where $L$ is the code length.

Considering complementary-correlation Golay $\operatorname{codes}^{6}$, they are by definition bipolar codes consisting in sequences of \pm 1 . Physical constrains have impeded the direct implementation of bipolar codes in systems based on intensity pulses; this limitation could be eventually overcome by modifying the bipolar Golay codes into unipolar (binary) pulse sequences ${ }^{6}$. Even though the unipolar Golay codes offers a lower coding gain with respect to bipolar Golay sequences (the coding gain of bipolar Golay is $C_{\text {gain }}=\sqrt{L}$, representing $3 \mathrm{~dB}$ more than the unipolar Golay codes) they have allowed the real implementation of the method in systems employing intensity pulses, such as OTDR and BOTDA sensors.

In order to obtain a higher SNR enhancement, we propose in this paper the use of bipolar sequences (such as the original bipolar Golay codes ${ }^{6}$ ) in BOTDA sensors, making simultaneous use of the Brillouin gain and Brillouin loss spectra. This method can be implemented using a dual-parallel optical modulator ${ }^{7}$, in which high carrier suppression can be achieved using a proper DC bias, providing also the possibility to select either the lower- or upper-frequency sideband (using another bias voltage ${ }^{7}$ ). Thus, every bit of the bipolar code is transmitted in one of the single sidebands according to the patterns defined by the bipolar codes. Figure 1(a) shows the optical SSB-SC modulated spectra obtained with a dualparallel Mach-Zehnder modulator (DP-MZM) when the intensity level of either the upper- or lower-frequency sideband is maximized. Considering that the probe signal is spectrally placed at the suppressed carrier frequency (see Fig. 1), i.e. at the nominal laser frequency, both single sidebands can be symmetrically generated at equal spectral distance from the probe frequency. In this way, bits ' 1 ' in the bipolar codes are generated using the upper-frequency sideband (inducing Brillouin gain in the probe), while bits ' -1 ' are in the lower-frequency sideband, inducing Brillouin loss. It is important to notice that in this configuration the pump-probe frequency detuning is maintained independently of the sign associated to the transmitted pulses, ensuring that the magnitudes of Brillouin gain and loss are indeed identical. Since the carrier of the SSB modulated pump and the probe signal counter-propagate in the fiber at the same frequency, carrier suppression is essential to avoid interference noise in measured BOTDA traces. Figure 1(b) shows an example of a pulse sequence corresponding to a 16-bit bipolar Golay codeword. The pulses at both lower- and upper-frequency sidebands and belonging to the same Golay sequence are illustrated by two different curves obtained by selective spectral filtering (in red and blue), to clearly identify the two frequencies involved in the implementation of the bipolar coded pump signal.
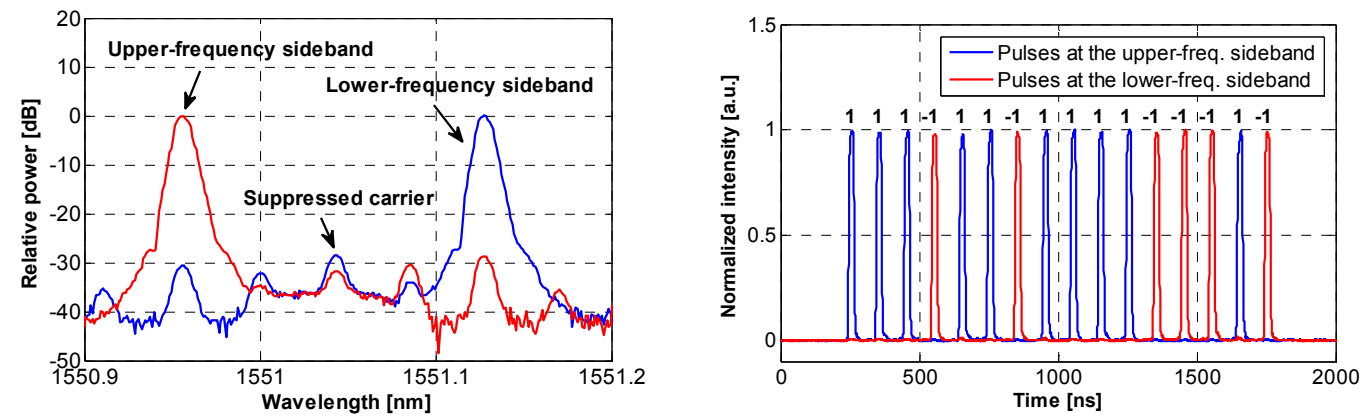

Figure 1. Principle of the SSB-SC modulation to generate a pump signal based on bipolar codes. (a) Spectra of the SSB-SC modulation obtained using a dual-parallel modulator. (b) Example of a single SSB-SC modulated 16-bit bipolar Golay codeword.

\section{EXPERIMENTAL SETUP}

The implemented BOTDA system using SSB-SC and bipolar coding is shown in Fig. 2. The light from a distributed feedback laser (DFB) at $1551 \mathrm{~nm}$ is split into pump and probe arms using an optical splitter. The probe signal is generated at the nominal laser frequency. A variable optical attenuator (VOA) has been used to adjust the probe power 
level at the fiber input, together with a polarization controller (PC) and a polarization switch (PS) to suppress the polarization dependence on the measured BOTDA traces. To generate the proper pump signal, the CW light from the laser is first SSB-SC modulated using a DP-MZM ${ }^{7}$. While the optical carrier is suppressed by the use of a proper DC bias, an arbitrary waveform generator (AWFG) provides another bias voltage to select the proper single-sideband according to the bipolar Golay codes. In order to scan the Brillouin frequency, a microwave generator is also connected to the DP-

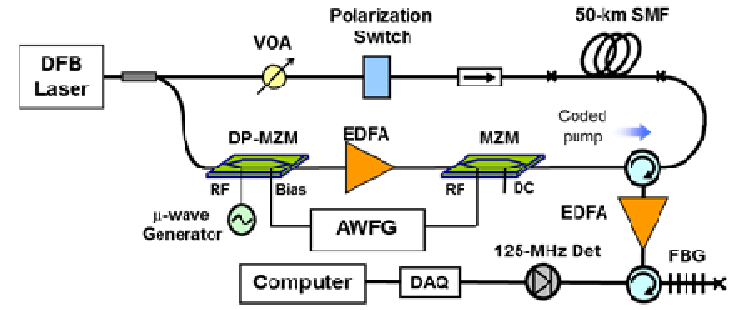

Figure 2. Experimental setup

MZM through a microwave splitter, which allows the injection of the in-phase and in-quadrature signals required for SSB modulation ${ }^{7}$. An Erbium-doped fiber amplifier (EDFA) is placed after the DP-MZM in order to increase the pump power launched into the fiber. A standard MZM is then used to generate synchronized RZ-intensity pulses, using $20 \mathrm{~ns}$ pulses (attaining $2 \mathrm{~m}$ spatial resolution) and $20 \%$ duty cycle. Pulses are then sent into a $50 \mathrm{~km}$ single mode fiber (SMF).

At the receiver, an EDFA operating in linear gain regime is used as a preamplifier. A narrowband fiber Bragg grating (FBG), centered at the probe frequency, is used to filter out the two Rayleigh components generated by the SSB-SC pulse sequences (upper and lower sidebands) and the amplified spontaneous emission (ASE) noise introduced by the preamplifier. Then, a $125 \mathrm{MHz}$ photodetector is connected to a fast data acquisition card (DAQ).

\section{EXPERIMENTAL RESULTS}

In order to validate the proposed SSB-SC technique and to evaluate the real SNR enhancement provided by bipolar pulses, the BGS along $50 \mathrm{~km}$ of fiber has been first measured employing bipolar Golay sequences with different code lengths. For every code length, the SNR levels of the decoded BOTDA traces have been compared with the ones obtained with single-pulse measurements (allowing for the estimation of the experimental coding gain) and with the SNR enhancement resulting from using conventional unipolar (binary) Golay codes. Figure 3 shows a comparison of the coding gain (experimental and theoretical), as a function of the code length, when using bipolar and unipolar Golay codes. Results indicate that the measured coding gains are in good agreement with the expected theoretical values ${ }^{6}$. It is evident from Fig. 3 that when bipolar Golay

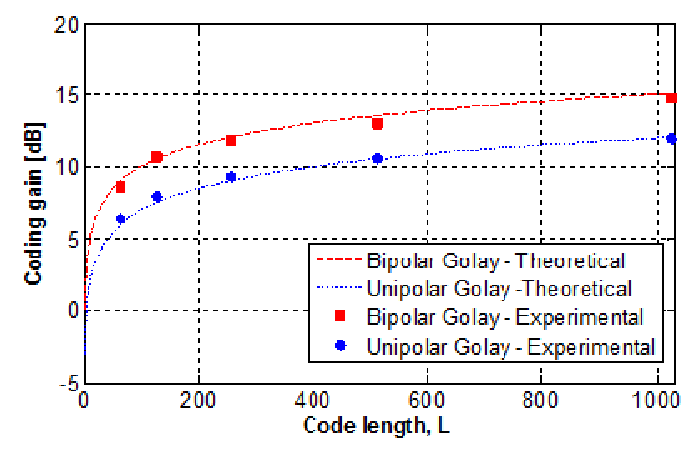

Figure 3. Experimental and theoretical coding gain for both unipolar ad bipolar Golay codes. codes are used in BOTDA systems, a higher SNR enhancement (3 $\mathrm{dB}$ more) is obtained when compared to the equivalent unipolar Golay sequences. This additional coding gain allows for a sensing distance extension by another $15 \mathrm{~km}$ in conditions similar to a unipolar Golay coding, while maintaining the same spatial and measurand resolutions.

Figure 4(a) shows the decoded BGS obtained with 128 bit bipolar Golay codes and 1000 time-averaged traces. The Brillouin linewidth has been measured to be around $60 \mathrm{MHz}$, as expected from $20 \mathrm{~ns}$ pump pulses. By fitting the decoded BGS, the BFS has been estimated as a function of the distance along the $50 \mathrm{~km}$ fiber, as depicted in Fig. 4(b). Calculating the standard deviation of the BFS, the frequency resolution has been found to be $1.0 \mathrm{MHz}$, corresponding to a temperature and strain resolution of $1{ }^{\circ} \mathrm{C}$ and $20 \mu \varepsilon$, respectively. Assuming that the maximum attainable sensing distance can be estimated using the SNR at the fiber end (which in this case is $12 \mathrm{~dB}$ at $50 \mathrm{~km}$ distance), a maximum sensing distance of $110 \mathrm{~km}$ can be potentially reached with $2 \mathrm{~m}$ spatial resolution (note that an SNR of $12 \mathrm{~dB}$ corresponds to an additional sensing range enhancement of $60 \mathrm{~km}$, resulting in a total distance of $110 \mathrm{~km}$ ).
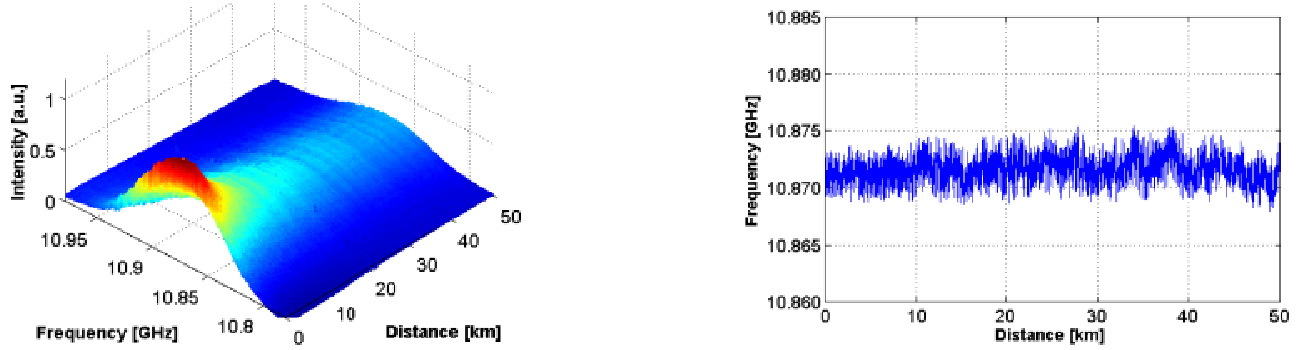

Figure 4. (a) Decoded BGS measured using 128-bit bipolar Golay codes and SSB-SC modulation. (b) Respective BFS profile. 
In addition to the larger SNR enhancement provided by bipolar Golay codes, both techniques (unipolar and bipolar coding) have also been compared in terms of pump depletion robustness. Under the same measurement conditions, BOTDA traces at the peak Brillouin gain have been measured with both bipolar and unipolar Golay codes, as shown in Fig. 5. We can clearly observe that, due to the uniform BFS (as reported in Fig. 4(b)), a continuous energy transfer from the pump to the probe takes place when unipolar Golay codes are used, inducing strong pump depletion levels. However, when using the proposed method based on SSB-SC pulses and bipolar codes, much lower levels of depletion can be observed. This is because the energy transferred from pulses in the upper-frequency sidebands (Brillouin gain process) are compensated by the energy transferred from the

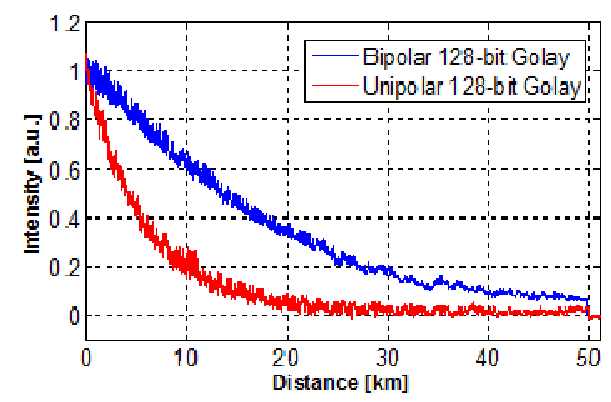

Figure 5. Decoded traces at the peak BGS with unipolar and bipolar Golay codes. probe to the pulses at the lower-frequency sideband. As a consequence, the power of the coded probe signal does not increase significantly, as in the case of unipolar coding; and therefore, limited levels of pump depletion or excess amplification occur. This issue is evident in Fig. 6(a), where we can compare the residual coded pump power after propagating along the $50 \mathrm{~km}$ sensing fiber with (red curve) and without (black curve) Brillouin interaction. It is actually worth noticing that pump pulses at the lower-frequency sideband are amplified along the fiber, while pulses at the upperfrequency sideband are depleted. Although this power unbalance occurs after propagating along a long sensing fiber, the intensity variations of the pulses are limited to about $2 \%$ of the pulse power measured with no Brillouin interaction. On the other hand, as reported in Fig. 6(b), when unipolar Golay codes are used (with the same involved optical power levels), the residual pump is clearly depleted up to $12 \%$ after propagating along the $50 \mathrm{~km}$ of fiber.
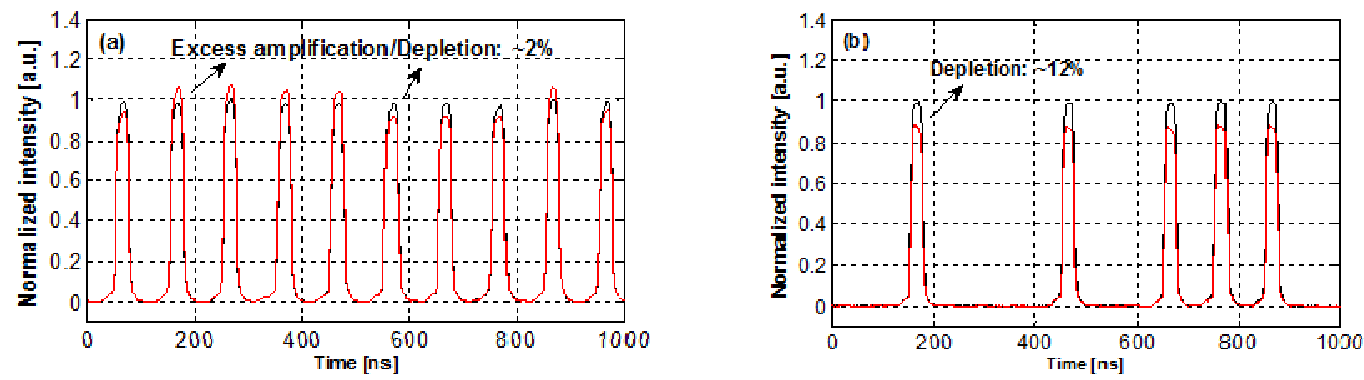

Figure 6. Residual pump after propagating along $50 \mathrm{~km}$ of fiber, when using (a) bipolar and (b) unipolar Golay codes. The black curves represent the residual power with no SBS interaction, while the red curves correspond to the case of maximum SBS gain/loss.

In conclusion, we have proposed a novel method to generate the pump signal in BOTDA sensors, which is based on SSB-SC modulation and bipolar codes. In particular we have demonstrated that the SNR enhancement obtained using complementary-correlation Golay codes is higher than the one attained with any other known coding method for Brillouin-based sensing. The proposed technique opens the possibility to use other types of bipolar sequences in BOTDA sensors, such as those ones used in radar systems. Thanks to its perfectly symmetric operation in gain and loss modes, stimulated Brillouin scattering offers the unique possibility to implement bipolar coding in distributed optical sensing.

\section{ACKNOWLEDGMENTS}

The authors would like to acknowledge the company OMNISENS (Switzerland), the Swiss National Foundation (Project NCCR-QP-IPP) and the Swiss Commission for Technology and Innovation (Project 13122.1) for their support.

\section{REFERENCES}

[1] Horiguchi, T. et al., J. Lightwave Technol. 13(7), 1296-1302, 1995.

[2] Angulo-Vinuesa, X. et al., J. Lightwave Technol. 30(8), 1060-1065, 2012.

[3] Soto, M. A., Bolognini, G., Di Pasquale, F., and Thévenaz, L., Opt. Lett. 35(2), 259-261, 2010.

[4] Liang, H., Li, W., Linze, N., Chen, L, and Bao, X., Opt. Lett. 35(10), 1503-1505, 2010.

[5] Soto, M. A., Bolognini, G., and Di Pasquale, F., Opt. Express 18(14), 14878-14892, 2010.

[6] Nazarathy, M., et al., J. Lightwave Technol. 7(1), 24-38, 1989.

[7] Kawanishi, T., and Izutsu, M., IEEE Photon. Tech. Lett. 16(6), 1534-1536 